
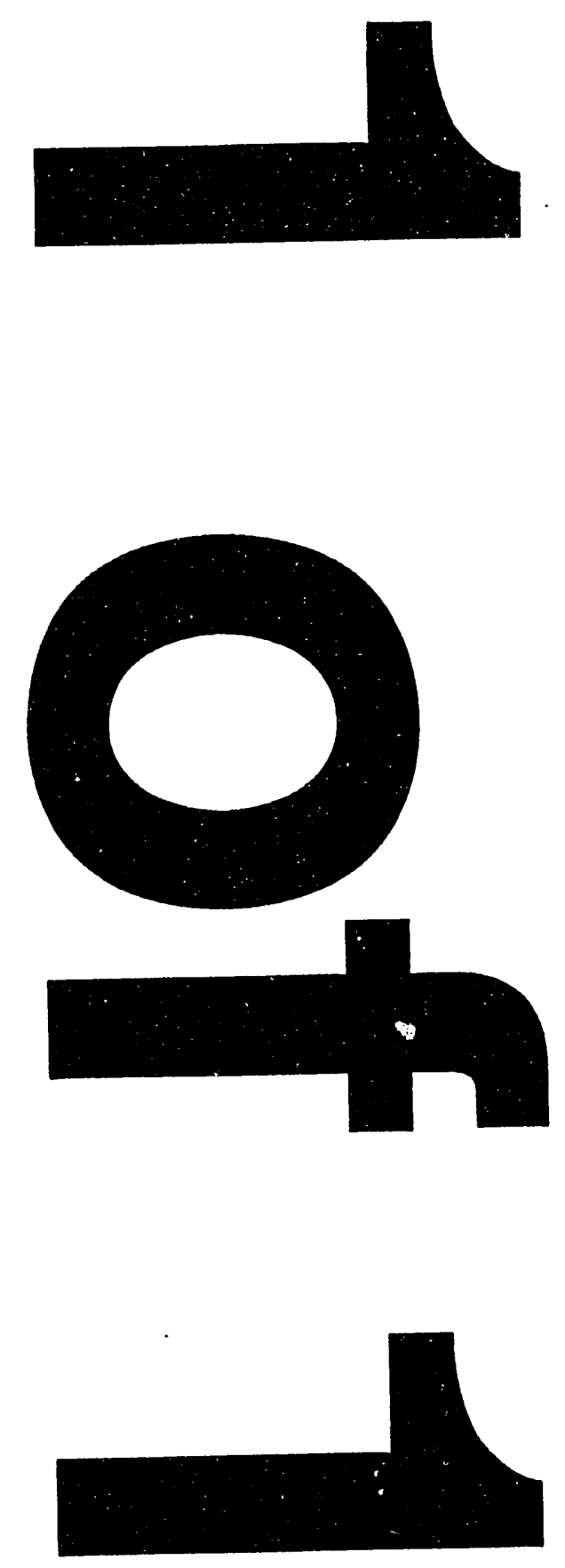
Technical Progress Report

\title{
Use of Cloud Observations and Mesoscale Meteorology Models to Evaluate and Improve Cloud Parameterizations
}

\author{
1 October 1992 - 30 September 1993
}

DOE Grant DE-FG02-92ER61364

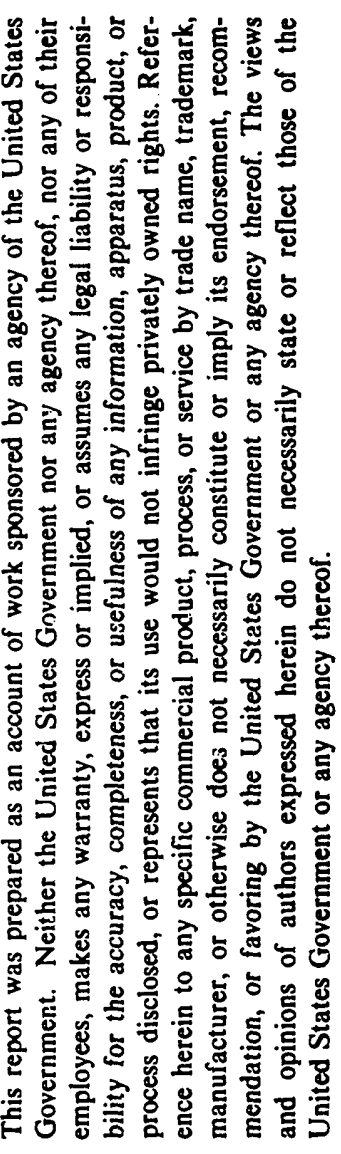

\author{
Prepared for: \\ Peter W. Lunn, Program Manager \\ Atmospheric Radiation Measurement Program \\ Environmental Sciences Division \\ Office of Health and Environmental Research \\ Office of Energy Research \\ Department of Energy \\ Washington, D. C. 20585
}

Prepared by:

Chris J. Walcek, Principal Investigator

Atmospheric Sciences Research Center

State University of New York at Albany 100 Fuller Rd.

Albany, NY 12205

(518) $442-3840$

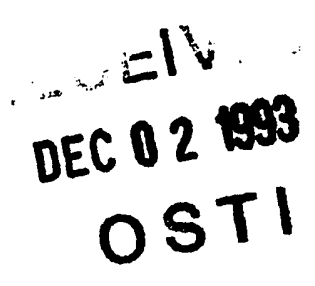

30 September 1993

\section{MASTER}




\section{Project Statement of Work}

This research program utilizes satellite and surface-derived cloud observations together with standard meteorological measurements to evaluate and improve our ability to accurately diagnose cloud coverage. Results of this research will be used to compliment existing or future parameterizations of cloud effects in general circulation models, since nearly all cloud parameterizations must specify a fractional area of cloud coverage when calculating radiative or dynamic cloud effects, and current parameterizations rely on rather crude cloud cover estimates.

During the first phase of this research program, our goal is to evaluate and improve the methods for calculating cloud cover within a mesoscale meteorology model. To accomplish this, a mesoscale meteorology model will be quantitatively evaluated using available cloud cover databases, including the U. S. Air Force 3DNEPH and RTNEPH satellite-derived cloud fields, as well as CART data as they become available. Hourly-averaged distributions of the modelderived cloud fields will be compared with observed clouds at the finest spatial and temporal resolution of the corresponding datasets. Algorithms currently used in global or mesoscale meteorological models to assess cloud coverage will be objectively evaluated. We will then carry out an innovative search for relationships between cloud coverage and numerous meteorological factors such as relative humidity, stability, wind snear, moisture convergence, precipitation rate and other parameters.

During the second phase of this research, the cloud cover data and improved parameterizations of cloud coverage developed during the first phase will be incorporated into a mesoscale meteorology model. Model forecasts which utilize the observed cloud coverage and depth should be improved relative to forecasts which crudely specify cloud properties. 


\section{Project Progress: 1 Oct. 1992 - 30 Sept. 1993}

During the second year of this research program, we have continued our analysis of cloud cover and its relationship with related meteorological parameters using the U. S. Air Force cloud cover archive. We have developed resolution-dependent algorithms that can calculate cloud cover at any level of the troposphere using the averaged relative humidity of the layer that agrees with cloud cover reported by surface observers, aircraft, and satellite observations archived in the U. S. Air Force 3DNEPH archive. We are currently evaluating these cloud cover algorithms using the incoming ARM CART shortwave radiation measurements, and are attempting to ascertain the general applicability of our previous studies. We find that cloud cover algorithms used by current climate models underpredict cloud cover under dry conditions, and our analysis of cloud cover measurements shows that cloud amount increases with increasing humidity at all relative humidities. We see no clear evidence that cloud cover vanishes at a "critical humidity". Based on these initial comparisons, we feel that current climate models cannot adequately assess the changes in cloud cover that may results from small changes in relative humidity in a modified climate. Thus, a potentially important feedback between climate change and changes in cloud cover are probably not adequately simulated by current models of global warming.

We find that a state-of-the-science mesoscale meteorology model has a tendency to overpredict relative humidity in the upper troposphere, and this overprediction will lead to overprediction of cloud cover. These areas of excessive relative humidity tend to occur in conditionally unstable areas where mean upward motion is occurring. Our initial hypothesis of the cause of this discrepancy centered around the convective mixing algorithm used to vertically redistribute moisture and heat within unstable atmospheric columns. However, subsequent testing of this hypothesis by not allowing any convective-scale mixing within the model did not significantly remove the tendency of the model to saturate the upper troposphere under unstable conditions. We are currently investigating other possible sources of this error which would lead to excessive cloud cover within climate models. 
An improved parameterization convective-scale vertical mixing has been tested using GATE measurements, and is also being evaluated with the incoming CART measurements. This standalone Single Column Model (SCM) will be used to assess the heating and moistening tendencies due to buoyantly-induced convection within hydrostatic models of the atmosphere, as well as precipitation rates attributable to subgrid-scale convective processes. The model combines an improved treatment of cloud-scale turbulent and microphysical processes within a detraining buoyant plume cloud model. We have adopted previous closure assumptions within the model, but find evidence for a new closure: an approximately fixed fraction of the mass of any unstable layer in the atmosphere leaves that layer per hour. This closure assumption was suggested after careful analysis of the GATE observations, and we are currently assessing incoming CART data to support or refute this observation.

In addition to the time devoted by Dr. Walcek to this research effort, two post-doctoral research associates and a graduate student are contributing to various technical aspects of this project.

Dr. Walcek presented results from this research effort at three scientific meetings: the Fourth Symposium on Global Change Studies held in Anaheim, California, 17-22 January 1993; the ARM science team meeting held in March 1993; and the 20th Conference on Hurricanes and Tropical Meteorology held in San Antonio, Texas from 10-14 May 1993. We are also revising a manuscript for journal publication summarizing the research results described above. Meeting abstract citations are listed at the end of this progress report.

\section{Plan for Next Year:}

1 Dec. 1993 - 30 Nov. 1994

During the next funding year of this research effort, the cloud cover data and improved parameterizations of cloud coverage will be incorporated into a mesoscale meteorology model. Model forecasts which utilize the observed cloud coverage and depth should be improved relative to forecasts which crudely specify cloud properties. During this period, we anticipate 
running improved versions of the MM4 meteorological model on DOE supercomputers, for which we have submitted applications for supercomputer resources. We plan to append incoming CART cloud cover and related meteorological data to our existing cloud observation database to expand the range of conditions under which we assess cloud cover, and hopefully utilize observations that contain a higher precision than the archived 3DNEPH data that currently composes the majority of the database.

As we develop our new convective mixing algorithm, we will begin including methods of assessing cloud cover from convective mass and moisture fluxes calculated by the model. We have found that in order to simulate precipitation accurately using our new convective parameterization, it is necessary to account for non-buoyantly induced (i. e. stable) precipitation processes. Measurements of precipitation cannot easily distinguish "stable" from "unstable" precipitation. However, models of buoyantly-induced convection cannot properly simulate "stable" precipitation, by definition. We have found that during the GATE convective measurements, we see precipitation is excess of what can be accounted for considering only convective processes, and therefore we hypothesize that some of this rain may result from "stable" processes not directly related with buoyant mechanisms.

Conference presentations, abstracts, and journal manuscripts resulting from this research effort:

Walcek, C. J., 1993: Factors influencing regional-scale cloud cover: Investigations using satellite-derived cloud cover and standard meteorological observations. Fourth Symposium on Global Change Studies, Anaheim, CA 17-22 January 1993. American Meteorological Society, 45 Beacon St., Boston, MA 02108, 235-236.

Walcek, C. J., 1993: A cumulus parameterization scheme of detraining drafts. $20^{\text {th }}$ Conference on Hurricanes and tropical meteorology, San Antonio, TX 10-14 May 1993. American Meteorological Society, 45 Beacon St., Boston, MA 02108, 345-348.

Walcek, C. J., 1993: Cloud cover and its relationship with relative humidity during a springtime midlatitude cyclone. Under revision, Monthly Weather Review. 

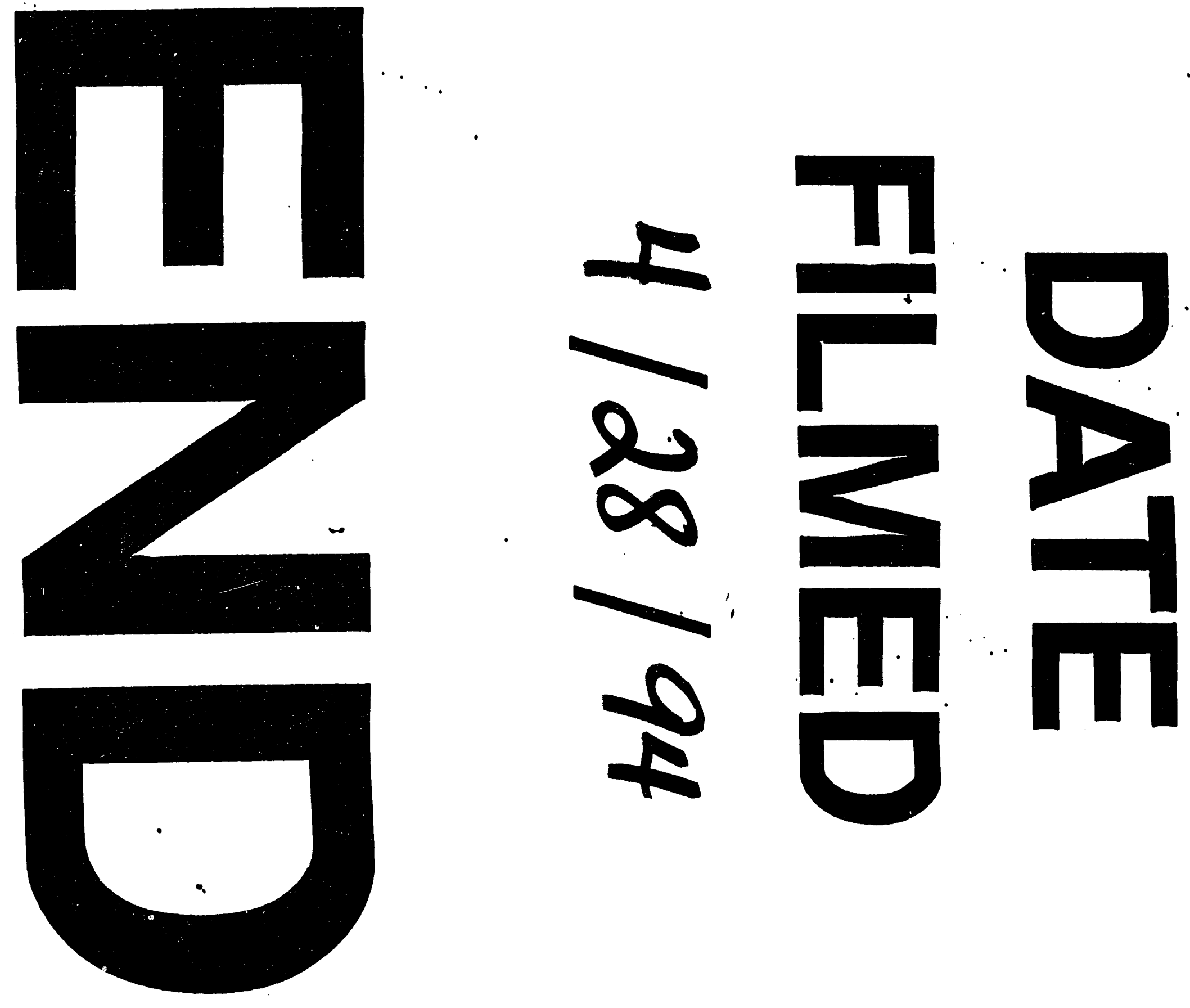